\title{
Über die Struktur stationärer zufälliger Funktionen
}

\author{
Von Kari Karhunen
}

In einer früheren Abhandlung (KARHUNEN [1]) haben wir eine einheitliche lineare Theorie der zufälligen Funktionen zu entwickeln versucht und einige Anwendungen auf stationäre zufällige Funktionen behandelt. Im folgenden werden wir die letztgenannten Fragen etwas tiefer untersuchen und dabei insbesondere die Resultate, die WoLD [1] und Kolmogorofr-[1,2] im Falle stationärer Folgen von zufälligen Grössen gewonnen haben, für stetige stationäre zufällige Funktionen verallgemeinern. Teilweise dieselben Probleme hat Hanner [1] mit einer anderen Methode ohne Zuhilfenahme der Spektraldarstellung behandelt.

Wo nicht anders gesagt wird, werden wir im folgenden der Terminologie und den Bezeichnungen unserer oben genannten Abhandlung folgen.

Diese Arbeit ist während unserem Aufenthalt als Dozentstipendiat an dem Institut für Versicherungsmathematik und Mathematische Statistik der Hochschule Stockholm durchgeführt worden. Es sei uns gestattet an dieser Stelle dem Vorgesetzten des Institutes, Herrn Prof. Dr. H. Cramér, unseren tief empfundenen Dank für seine unermüdliche Hilfsbereitschaft und für manche wertvolle Ratschläge auszusprechen.

\section{§ 1. Untergeordnete zufällige Funktionen}

1. Es sei $x(t)$ eine zufällige Funktion und $L_{2}(x)$ der zu ihr gehörende lineare Raum (vgl. Karhunen [1], S. 26). Eine andere zufällige Funktion $y(u)$ heisst $x(t)$ (linear) untergeordnet, wenn $L_{2}(y) \subseteq L_{2}(x)$. Gilt insbesondere $L_{2}(y)=L_{2}(x)$ heissen $x(t)$ und $y(u)$ (linear) zusammengeordnet.

Ist insbesondere $x(t)$ stationär (vgl. KARHunen [1], S. 54), ist $y(t)$, welche wie $x(t)$ auf der reellen Zahlengerade definiert ist, ihr untergeordnet (bzw. zusammengeordnet), und sind $x(t)$ und $y(t)$ stationär korreliert, $\mathrm{d}$. h. ist

$$
r_{x y}(s-t)=E x(s) \overline{y(t)}
$$

eine Funktion von $s-t$ allein, so sagen wir, dass $y(t)$ der zufälligen Funktion $x(t)$ stationär untergeordnet (bzw. zusammengeordnet) ist.

Wir bezeichnen mit $L_{2}(x ; t)$ die geschlossene lineare Hülle der Menge $\{x(s)$, $s \leqq t\}$. Ist $y(t)$ zu $x(t)$ stationär untergeordnet (bzw. zusammengeordnet) und gilt dazu $L_{2}(y ; t) \subseteq L_{2}(x ; t)$ (bzw. $L_{2}(y ; t)=L_{2}(x ; t)$ ) für alle $t$, so heisst die Unterordnung (bzw. Zusammenordnung) gleichförmig. 
K. KARHUNEN, Über die Struktur stationärer zufälliger Funktionen

2. Bekanntlich (CRAMḱr [2]) kann eine stetige stationäre zufällige Funktion $x(t)$ in der Form

$$
x(t)=\int_{-\infty}^{+\infty} e^{i \lambda t} d Z_{x}(\lambda)
$$

dargestellt werden, wo $Z_{x}$ eine mit $x(t)$ zusammengeordnete zufällige Spektralfunktion ist (vgl. Karhunen [1]). Für die Korrelationsfunktion $r_{x x}(t)=$ $=E x(s+t) \overline{x(s)}$ von $x(t)$ gilt die entsprechende Darstellung (KHintchine [1])

$$
r_{x x}(t)=\int_{-\infty}^{+\infty} e^{i \dot{\alpha} t} d F_{x}(\lambda)
$$

wo $F_{x}$ beschränkt und nichtabnehmend ist und der Bedingung $\Delta F_{x}=\left\|\Delta Z_{x}\right\|^{2}$ genügt.

Jedes Element $z$ in $L_{2}\left(Z_{x}\right)=L_{2}(x)$ kann in der Form

$$
z=\int_{-\infty}^{+\infty} f_{z}(\lambda) d Z_{x}(\lambda)
$$

dargestellt werden, wo $f_{z}$ eine in bezug auf $\boldsymbol{F}_{x}$ quadratisch integrierbare komplexe Funktion ist (Karhunen [1], Satz 9). Wenn wir insbesondere eine $x(t)$ stationär untergeordnete zufällige Funktion $y(t)$ betrachten, gibt es also für jedes $t$ eine Funktion $f(\lambda ; t)$ von $\lambda$ so, dass

$$
y(t)=\int_{-\infty}^{+\infty} f(\lambda ; t) d Z_{x}(\lambda)
$$

Aus (1), (2) und (4) folgt für beliebige $s$ und $t$

$$
\begin{aligned}
E x(s) \overline{y(t)}=\int_{-\infty}^{+\infty} e^{i \lambda s} \overline{f(\lambda ; t)} d F_{x}(\lambda)=E x(s-t) \overline{y(0)}= \\
=\int_{-\infty}^{+\infty} e^{i \lambda(s-t)} \overline{f(\lambda ; 0)} d F_{x}(\lambda)
\end{aligned}
$$

und somit

$$
\int_{-\infty}^{+\infty} e^{i \lambda s} \overline{\left[f(\lambda ; t)-e^{i \dot{i} t} f(\lambda ; 0]\right.} d F_{x}(\lambda) \equiv 0 .
$$

Dies ist aber möglich nur, wenn für jedes $t$ fast überall in bezug auf $\boldsymbol{F}_{x}$ (d. h. für alle $\lambda$ ausserhalb einer Menge $M$ über welche die Variation von $F_{x}$ verschwindet)

$$
f(\lambda ; t)=e^{i \lambda t} f(\lambda ; 0)
$$

gilt. Bezeichnen wir noch $f(\hat{\lambda} ; 0)=\gamma_{y}(\lambda)$, bekommen wir unmittelbar den folgenden 
Satz 1: Ist $x(t)$ eine stetige stationäre zufällige Funktion und $y(t)$ ihr stationär untergeordnet, so gibt es eine in bezug auf $F_{x}$ quadratisch integrierbare, fast überall in bezug auf $\boldsymbol{F}_{x}$ eindeutig bestimmte komplexe Funktion $\gamma_{y}(\lambda)$ so, dass

$$
y(t)=\int_{-\infty}^{+\infty} e^{i \lambda t} \gamma_{y x}(\lambda) d Z_{x}(\lambda) .
$$

Insbesondere ist also $y(t)$ stationär und stetig und es gilt mit entsprechenden Bezeichnungen wie in (2) und (3)

$$
\begin{gathered}
Z_{y}(S)=\int_{S} \gamma_{y x}(\lambda) d Z_{x}(\lambda), \\
F_{y}(\lambda)=\int_{-\infty}^{\lambda}\left|\gamma_{y x}(\lambda)\right|^{2} d F_{x}(\lambda), \\
r_{y x}(t)=\int_{-\infty}^{+\infty} e^{i \lambda t} \gamma_{y x}(\lambda) d F_{x}(\lambda) .
\end{gathered}
$$

Aus (6) folgt, dass $L_{2}\left(Z_{x}\right)=L_{2}\left(Z_{y}\right)$, d. h. $L_{2}(x)=L_{2}(y)$ dann und nur dann gilt, wenn fast überall in bezug auf $F_{x} \gamma_{y x}(\lambda)$ von Null verschieden ist (vgl. KaRhunen [1], S. 50). Dann und nur dann sind also $x(t)$ und $y(t)$ stationär zusammengeordnet.

3. Ist $y(t)$ stationär und stetig und mit $x(t)$ stationär korreliert, aber nicht notwendig $x(t)$ untergeordnet, so ist die Projektion von $y(t)$ auf $L_{2}(x)$

$$
P_{L_{z}(x)} y(t)=y_{x}(t)
$$

wegen $E x(s) \overline{y_{x}(t)}=E x(s) \overline{y(t)}$ zu $x(t)$ stationär untergeordnet. Weil $y(t)$ und $y_{x}(t)$ stationär sind und $y_{x}(s) \perp y(t)-y_{x}(t)$, ist $y(t)-y_{x}(t)$ stationär und man bekommt

$$
\begin{gathered}
y_{x}(t)=\int_{-\infty}^{+\infty} e^{i \lambda t} \gamma_{y x}(\lambda) d Z_{x}(\lambda), \\
y(t)-y_{x}(t)=\int_{-\infty}^{+\infty} e^{i \lambda t} d Z_{y-y_{x}}(\lambda),
\end{gathered}
$$

wo $L_{2}\left(Z_{y-y_{x}}\right) \perp L_{2}\left(Z_{x}\right)$. Es folgt daraus nach (7) und (8)

$$
r_{x y}(t)=\int_{-\infty}^{+\infty} e^{i \lambda t} d F_{x y}(\lambda)
$$

und

$$
r_{y y}(t)=\int_{-\infty}^{+\infty} e^{i \lambda t} d F_{y}(\lambda)
$$


K. KARHUNeN, Über die Struktur stationärer zufälliger Funktionen

mit

$$
F_{x y}(\lambda)=\int_{-\infty}^{\lambda} \overrightarrow{\gamma_{y x}} \overline{(\lambda)} d F_{x}(\lambda)
$$

und

$$
F_{y}(\lambda)=\int_{-\infty}^{\dot{\delta}}\left|\gamma_{\nu x}(\lambda)\right|^{2} d F_{x}(\lambda)+F_{y-\nu_{x}}(\lambda)
$$

Die Schwarzsche Ungleichung gibt wegen $\Delta F_{y-y_{x}}(\lambda) \geqq 0$

$$
\left|\Delta F_{x y}\right|^{2}=\left|\int_{\Delta} \gamma_{y x}(\lambda) d F_{x}(\lambda)\right|^{2} \leqq \int_{\Delta}\left|\gamma_{y x}(\lambda)\right|^{2} d F_{x}(\lambda) \int_{\Delta} d F_{x}(\lambda) \leqq \Delta F_{x} \Delta F_{y}
$$

$F_{x y}$ ist also eine Funktion von beschränkter Schwankung, deren Differenzen der Ungleichung

$$
\left|\Delta F_{x y}\right|^{2} \leqq \Delta F_{x} \Delta F_{y}
$$

genügen. Umgekehrt folgt aus (9) und (10), dass für beliebige beschränkte und nichtabnehmende reelle Funktionen $F_{x}$ und $F_{y}$ und eine komplexe Funktion $F_{x y}$ die der Ungleichung (15) genügt, immer zwei stetige stationäre und stationär korrelierte zufällige Funktionen $x(t)$ und $y(t)$ konstruiert werden können so, dass (3), (11) und (12) gelten. Man braucht nur

$$
\gamma_{y x}=\frac{d \overline{F_{x y}}}{d \boldsymbol{F}_{x}}, \quad \boldsymbol{F}_{y-y_{x}}=\boldsymbol{F}_{y}-\int_{-\infty}^{\dot{i}}\left|\gamma_{y x}(\lambda)\right|^{2} d \boldsymbol{F}_{x}(\lambda)
$$

definieren. Aus (15) folgt dann, dass $F_{y-y_{x}}$ nicht abnehmend ist, so dass man zwei orthogonale zufällige Spektralfunktionen $Z_{x}$ und $Z_{y-y_{x}}$ so geben kann, dass $\left\|\not Z_{x}\right\|^{2}=\Delta F_{x},\left\|\cdot \Delta Z_{y-y_{x}}\right\|^{2}=\Delta F_{y-y_{x}}$. Dieses Resultat, das leicht auch für beliebig viele stationäre zufällige Funktionen erweitert werden kann, hat Cramḱr [1] mit einer anderen Methode bewiesen. (Für stationäre Folgen, vgl. auch KoLmoGOROFF [1], § 3).

4. $y(t)$ heisst eine stationäre Komponente von $x(t)$, wenn $y(t)$ zu $x(t)$ stationär untergeordnet ist und $y(s) \perp x(t)-y(t)$ für alle $s$ und $t$.

Satz 2: $y(t)$ ist dann und nur dann eine stationäre Komponente von $x(t)$, wenn fast überall in bezug auf $F_{x}$

$$
\gamma_{y x}(\lambda)=e_{s}(\lambda)
$$

wo $e_{s}(\lambda)$ die charakteristische Funktion einer messbaren Menge $S$ ist (d. h. $e_{z}(\lambda)=1$ für $\lambda \in S$, sonst $e_{y}(\lambda)=0$ ). - Man kann sagen, dass jede Zerlegung einer stetigen stationären zufälligen Funktion in stationäre Komponenten einer Zerlegung ihres Spektrums in komplementäre Teilspektra entspricht. 
Beweis: Aus (5) und $x(t)-y(t) \perp L_{2}(y)$ folgt

$$
\int_{-\infty}^{+\infty} e^{i \lambda t}\left(1-\gamma_{y x}(\lambda) \overline{\gamma_{y x}(\lambda)} d F_{x}(\lambda)=0\right.
$$

für alle $t$ und somit

$$
\left(1-\gamma_{y x}(\lambda) \overline{\gamma_{y}(\lambda)}=0,\right.
$$

d. h. entweder $\gamma_{y x}(\lambda)=0$ oder $\gamma_{y x}(\lambda)=1$, fast überall in bezug auf $\boldsymbol{F}_{x}$. Weil $\gamma_{y x}(\lambda)$ eine messbare Funktion ist, ist diejenige Menge $S$, in welcher $\gamma_{y}(\lambda)=1$, messbar, woraus die Behauptung folgt.

\section{§ 2. Deterministische und indeterministische stationäre zufällige Funktionen}

5. Es sei fortwährend $x(t)$ eine stetige stationäre zufällige Funktion. Für $s \leqq t$ gilt dann offenbar $L_{2}(x ; s) \subseteq L_{2}(x ; t)$. Wenn $t \rightarrow-\infty$, konvergiert somit $L_{2}(x ; t)$ gegen einen Unterraum in $L_{2}(x)$, den wir mit $L_{2}(x ;-\infty)$ bezeichnen. Ist $L_{2}(x ;-\infty)=L_{2}(x)$, d. h. ist für jedes $t L_{2}(x ; t)=L_{2}(x)$, ist jedes $x(t)$ ein Element in $L_{2}(x ; s)$, wo $s$ beliebig gewählt werden kann. Das bedeutet, dass die zufällige Funktion $x(t)$ schon durch ihre Werte für $t \leqq s$ eindeutig für alle Werte des Arguments bestimmt wird. Die zufällige Funktion wird dann deterministisch genannt: Der andere extreme Fall ist, dass $L_{2}(x ;-\infty)$ nur das Nullelement enthält. Dann kann man sagen, dass die Werte von $x(t)$ für $t \leqq s$ beliebig wenig Auskunft über die Werte von $x(t)$ für $t \geqq u$ geben, wenn $u-s$ gross genug wird. Wir sagen, dass die zufällige Funktion dann rein indeterministisch ist.

Im allgemeinen ist natürlich $0 \subset L_{2}(x ;-\infty) \subset L_{2}(x)$. Man sieht leicht, dass dann $x(t)$ eindeutig in zwei stationäre Komponenten so zerlegt werden kann, dass die eine Komponente deterministisch, die zweite rein indeterministisch ist. In der Tat is die Projektion $x_{d}(t)$ von $x(t)$ auf $L_{2}(x ;-\infty)$, die wegen $L_{\mathbf{2}}(x ;-\infty) \subseteq L_{2}(x)$ zu $x(t)$ untergeordnet ist, eine stationäre Komponente von $x(t)$. Denn wir haben

$$
\begin{aligned}
& E\left(x_{d}(s) \overline{x(t)}\right)=E\left(P_{L_{2}(x ;-\infty)} x(s) \overline{x(t)}\right)=E\left(T_{h} P_{L_{s}(x ;-\infty)} x(s) \overline{T_{h} x(t)}\right)= \\
& =E\left(P_{r_{h} L_{2}(x ;-\infty)} x(s+h) \overline{x(t+h)},\right.
\end{aligned}
$$

wo $T_{h}$ diejenige lineare Transformation in $L_{2}(x)$ bedeutet; die jedes $x(t)$ in $x(t+h)$ überführt (vgl. KARHUNEN [1], S. 55). Nun ist aber offenbar $T_{h} L_{2}(x ;-\infty)=L_{2}(x ;-\infty)$, so dass wir

$$
E\left(x_{d}(s) \overline{x(t)}\right)=E\left(x_{d}(s+h \overline{x(t+h)})\right.
$$

bekommen, und $x_{d}(t)$ somit mit $x(t)$ stationär korreliert ist. Wegen $L_{2}(x ;-\infty)=$ $=P_{L_{2}(x ;-\infty)} L_{2}(x)=L_{2}\left(x_{d}\right)$ und $L_{2}\left(x_{d} ;-\infty\right)=P_{L_{2}(x ;-\infty)} L_{2}\left(x_{d}\right)=L_{2}\left(x_{d}\right)$ ist 


\section{K. KARHUNEN. Über die Struktur stationärer zufälliger Funktionen}

schliesslich $x_{d}(t)$ deterministisch. Aus $L_{2}(x ;-\infty) \ominus L_{2}\left(x_{d}\right)=0$ folgt nun sofort, dass $x(t)-x_{d}(t)$ rein indeterministisch ist.

Die Begriffe "deterministisch" und "rein indeterministisch" verdanken wir WoLD [1] für stationäre Folgen; er nennt die betreffenden Komponenten singulär bzw. regulär. Die Benennung »deterministisch» hat Dоoв [1] eingeführt. Wold [1] und KolmogorofF [1] haben gezeigt, dass für stationäre Folgen eine Zerlegung in eine deterministische und eine rein indeterministische Komponente möglich ist.

6. Es sei $h$ eine beliebige positive Zahl. Wir bezeichnen

$$
x(t ; h)=x(t)-P_{L_{v}(x ; t-h)} x(t) .
$$

Offenbar ist $x(t ; h)$ zu $x(t)$ stationär untergeordnet (aber im allgemeinen keine stationäre Komponente von $x(t)$, weil $L_{2}(x ; t-h)$ von $t$ abhängt und somit $x(t)$ nicht zu $x(s)-x(s ; h)$ orthogonal zu sein braucht). Ist $x(t)$ deterministisch, verschwindet $x(t ; h)$ identisch. Umgekehrt, wenn $x(t ; h)$ für jedes $h$ identisch verschwindet, ist $x(t)=P_{L_{3}(x ; t-h)} x(t)$ für jedes $h$, d. h. $x(t)=P_{L_{3}(x ;-\infty)} x(t)$ und somit $x(t)$ deterministisch.

Nach dem Satz 1 gibt es eine in bezug auf $\boldsymbol{F}_{\boldsymbol{x}}$ quadratisch integrierbare Funktion $\gamma(\lambda ; h)$ so, dass

$$
x(t ; h)=\int_{-\infty}^{+\infty} e^{i \lambda t} \gamma(\lambda ; h) d Z_{x}(\lambda) .
$$

Für $s-t>h$ ist $E x(s ; h) \overline{x(t ; h)}=0$, weil $x(t ; h)$ ein Element in $L_{2}(x ; t)$ und wegen $t<s-h$ auch in $L_{2}(x ; s-h)$ ist, während $x(s ; h)$ wegen (17) zu $L_{2}(x ; s-h)$ orthogonal ist. Aus (18) und (7) folgt somit

$$
\int_{-\infty}^{+\infty} e^{i \lambda t}|\gamma(\lambda ; h)|^{2} d F_{x}(\lambda)=0 \text { für }|t|>h .
$$

Man schliesst daraus, dass die Funktion

$$
\int_{-\infty}^{\lambda}|\gamma(\lambda ; h)|^{2} d F_{x}(\lambda)
$$

totalstetig ist und demnach, weil die Ableitung $F_{x}^{\prime}(\lambda)$ der monotonen Funktion $F_{x}(\lambda)$ fast überall existiert,

$$
\int_{-\infty}^{\lambda}|\gamma(\lambda ; h)|^{2} d F_{x}(\lambda)=\int_{-\infty}^{\lambda}|\gamma(\lambda ; h)|^{2} F_{x}^{\prime}(\lambda) d \lambda .
$$

Bekanntlich lässt sich $F_{x}(\lambda)$ als Summe von drei Funktionen

$$
F_{x}(\lambda)=F_{x}^{(1)}(\lambda)+F_{x}^{(2)}(\lambda)+F_{x}^{(3)}(\lambda)
$$


schreiben, die sämtlich nichtabnehmend sind, und zwar so, dass $F_{x}^{(1)}(\lambda)$ totalstetig ist und fast überall $F_{x}^{\prime}(\lambda)=F_{x}^{(1) \prime}(\lambda)$ gilt, $F_{x}^{(2)}(\lambda)$ eine reine Sprungfunktion ist und $F_{x}^{(3)}(\lambda)$ stetig ist. Die Ableitungen von $F_{x}^{(2)}(\lambda)$ und $F_{x}^{(3)}(\lambda)$ verschwinden ausserhalb einer Nullmenge $M$. Ist $M_{2}$ die abzählbare Menge der Unstetigkeitstellen von $F_{x}(\lambda), M_{3}=M-M_{2}$ und $M_{1}=R-M$, wo $R$ die ganze reelle Achse bedeutet, so gilt offenbar für jede messbare Menge $S$

$$
\int_{S} d F_{x}^{(v)}(\lambda)=\int_{S \cdot M_{v}} d F_{x}(\lambda) \quad(y=1,2,3)
$$

Wir können jetzt drei zufällige Spektralfunktionen durch

$$
Z_{x}^{(v)}(S)=\int_{S \cdot M_{y}} d Z_{x}(\lambda) \quad(v=1,2,3)
$$

so definieren, dass $\left\|\Delta Z_{x}^{(\nu)}\right\|^{2}=\Delta F_{x}^{(v)}$ und

$$
Z_{x}(S)=Z_{x}^{(1)}(S)+Z_{x}^{(2)}(S)+Z_{x}^{(3)}(S)
$$

$L_{2}\left(Z_{x}^{(1)}\right), L_{2}\left(Z_{x}^{(2)}\right)$ und $L_{2}\left(Z_{x}^{(3)}\right)$ sind zueinander orthogonal. Schreiben wir

$$
x_{i}(t)=\int_{-\infty}^{+\infty} e^{i \lambda t} d Z_{x}^{(v)}(\lambda)
$$

so ist

$$
x(t)=x_{1}(t)+x_{2}(t)+x_{3}(t)
$$

nach dem Satz 2 eine Zerlegung von $x(t)$ in drei stationäre Komponenten. Wir sagen (vgl. KARHUNEN [1], S. 70), dass $x_{1}(t)$ ein totalstetiges Spektrum, $x_{2}(t)$ ein Punktspektrum und $x_{3}(t)$ ein singuläres Spehtrum besitzt.

Weil $x_{1}(t), x_{2}(t)$ und $x_{3}(t)$ zueinander orthogonal sind, folgt aus (17)

$$
x(t ; h)=x_{1}(t ; h)+x_{2}(t ; h)+x_{3}(t ; h) .
$$

Nach (18) und (20) verschwindet $x(t ; h)$ identisch, wenn $F_{x}^{\prime}(\lambda)$ fast überall verschwindet. In diesem Fall ist also $x(t)$ sicher deterministisch., Es folgt daraus insbesondere, dass die Komponenten $x_{2}(t)$ und $x_{3}(t)$ deterministisch sind. Wir haben somit

$$
x(t ; h)=x_{1}(t ; h)=\int_{-\infty}^{+\infty} e^{i \lambda t} \gamma(\lambda ; h) d Z_{x}^{(1)}(\lambda) .
$$

Es bedeutet also keine wesentliche Einschränkung, wenn wir im folgenden der Einfachheit halber annehmen, dass $x(t)$ ein totalstetiges Spektrum hat.

Nach (19) haben wir.

$$
\int_{-\infty}^{+\infty} e^{i \lambda t}|\gamma(\lambda ; h)|^{2} E_{x}^{\prime}(\lambda) d \lambda=0 \text { für }|t|>h .
$$


K. KARHUNEN, Über die Struktur stationärer zufälliger Funktionen

Aus $x(s ; h) \perp L_{2}(x ; s-h)$ und $x(t) \in L_{2}(x ; s-h)$ für $t \leqq s-h$ folgt nach (8) weiter

$$
\int_{-\infty}^{+\infty} e^{i \lambda t} \gamma(\lambda ; h) F_{x}^{\prime}(\lambda) d \lambda=0 \text { für } t>h
$$

Die Funktionen $|\gamma(\lambda ; h)|^{2} F_{x}^{\prime}(\lambda)$ und $\gamma(\lambda ; h) F_{x}^{\prime}(\lambda)$ sind beide über $(-\infty,+\infty)$ absolut integrierbar (für $\gamma(\lambda ; \boldsymbol{h}) \boldsymbol{F}_{x}^{\prime}(\lambda)$ folgt dies sofort wegen der absoluten Integrierbarkeit von $F_{x}^{\prime}(\lambda)$ aus der Schwarzschen Ungleichung). Nach einem Satz von Hille und Tamarkin [1] kann die Fouriersche Transformierte einer absolut integrierbaren Funktion $f:(\lambda)$ auf einer Halbachse nur verschwinden, wenn das Integral

$$
\int_{-\infty}^{+\infty} \frac{|\log | f(\lambda)||}{1+\lambda^{2}} d \lambda
$$

konvergiert. Aus (27) und (28) folgt somit

$$
\int_{-\infty}^{+\infty} \frac{\left|\log \left[|\gamma(\lambda ; h)|^{2} F_{x}^{\prime}(\lambda)\right]\right|}{1+\lambda^{2}} d \lambda<\infty, \int_{-\infty}^{+\infty} \frac{\left|\log \left[|\gamma(\lambda ; h)| F_{x}^{\prime}(\lambda)\right]\right|}{1+\lambda^{2}} d \lambda<\infty .
$$

Nun ist aber

$$
\begin{aligned}
0 & \leqq\left|\log F_{x}^{\prime}\right|=\left.|2 \log | \gamma\left|+2 \log F_{x}^{\prime}-\log \right| \gamma\right|^{2} F_{x}^{\prime}|\leqq 2| \log |\gamma| F_{x}^{\prime} \mid+ \\
& +\left.|\log | \gamma\right|^{2} F_{x}^{\prime} \mid, \\
0 & \leqq|\log | \gamma||=|\log | \gamma\left|F_{x}^{\prime}-\log F_{x}^{\prime}\right| \leqq|\log | \gamma\left|F_{x}^{\prime}\right|+\left|\log F_{x}^{\prime}\right|,
\end{aligned}
$$

so dass die Bedingungen (29) mit den folgenden äquivalent sind:

$$
\int_{-\infty}^{+\infty} \frac{\left|\log F_{x}^{\prime}(\lambda)\right|}{1+\lambda^{2}} d \lambda<\infty, \int_{-\infty}^{+\infty} \frac{|\log | \gamma(\lambda ; h)||}{1+\lambda^{2}} d \lambda<\infty .
$$

Ist die erste Bedingung (29)' nicht erfüllt, kann eine von Null verschiedene Funktion $\gamma(\lambda ; h)$ nicht existieren. Wir haben also zunächst den

Satz 3: Wenn das Integral

$$
\int_{-\infty}^{+\infty} \frac{\left|\log F_{x}^{\prime}(\lambda)\right|}{1+\lambda^{2}} d \lambda
$$

divergiert, ist die zufällige Funktion $x(t)$ deterministisch. Insbesondere ist $x(t)$ deterministisch, wenn $F_{x}^{\prime}(\lambda)$ in einer Menge von positivem Mass verschwindet.

Es sei bemerkt, dass wegen $\log F_{x}^{\prime}<F_{x}^{\prime \prime}$ nur kleine Werte von $F_{x}^{\prime}$ die Divergenz des Integrals (30) verursachen können. 


\section{§ 3. Die kanonische Darstellung einer indeterministischen stationären zufälligen Funktion}

7. Wir nehmen jetzt an, dass das Spektrum von $x(t)$ totalstetig ist und dass das Integral (30) konvergiert. Wir wissen dann, dass $x(t)$ sich in der Form

$$
x(t)=\int_{0}^{\infty} g(u) d_{u} \xi(t-u)=\int_{-\infty}^{t} g(t-u) d \xi(u)
$$

darstellen lässt, wo $\xi(S)$ eine für alle Lebesgue-messbare Mengen auf der reellen Achse definierte zufällige Spektralfunktion ist, die dem LEbEsGuEschen Mass $m$ entspricht, $d . h$. der Bedingung

$$
\|\xi(S)\|^{2}=m(S)
$$

genügt (KARHUNEN [1], Satz 16). $x(t)$ und $\xi(S)$ sind zusammengeordnet.

Eine zufällige Spektralfunktion mit der Eigenschaft (32) wird homogen genannt. Sie hat Eigenschaften, die sie aufs engste mit stationären zufälligen Funktionen zusammenknüpfen. In der Tat: wird mit $T_{k}$ eine Translation der reellen Achse bezeichnet, die jeden Punkt $t$ in den Punkt $t+h$ führt, so ist offenbar $\xi\left(T_{t} S\right)$ eine stationäre zufällige Funktion von $t$. Ist insbesondere $S$ ein Intervall $(a, b)$, bekommat man für $\xi\left(T_{t} S\right)$ die Spektraldarstellung

$$
\xi\left(T_{t} S\right)=\xi(a+t, b+t)=\frac{1}{\sqrt{2 \pi}} \int_{-\infty}^{+\infty} e^{i \lambda t} \frac{e^{i \dot{\lambda} b}-e^{i \lambda a}}{i \lambda} d \xi^{*}(\lambda)
$$

wo $\xi^{*}$ wieder eine homogene zufällige Spektralfunktion ist (vgl. KarHunen [1], S. 53). Allgemeiner gilt

$$
\xi(S)=\int_{-\infty}^{+\infty}\left\{\frac{1}{\sqrt{2 \pi}} \int_{S} e^{i, t} d t\right\} d \xi^{*}(\lambda)
$$

und umgekehrt

$$
\xi^{*}(S)=\int_{-\infty}^{+\infty}\left\{\frac{1}{\sqrt{2 \pi}} \int_{S} e^{-i \lambda t} d \lambda\right\} d \xi(t)
$$

Der Beweis dieser Formeln ist demjenigen von (33) vollkommen analog. Wir nennen $\xi^{*}$ die Fouriarsche Transformierte von $\xi$. Wegen (34) und (35) sind $\xi$ und $\xi^{*}$ offenbar zusammengeordnet.

Satz 4: Ist $\xi(S)$ eine homogene zufällige Spektralfunktion und $f(t)$ eine quadratisch integrierbare komplexe Funktion und werden ihre Founienschen Transformierten mit $\xi^{*}(S)$ bzw. mit $f^{*}(t)$ bezeichnet, so gilt die Parsevalsche Formel 
K. KARHUNEN, Über die Struktur stationäreer zufälliger Funktionen

$$
\int_{-\infty}^{+\infty} f(t) d \xi(t)=\int_{-\infty}^{+\infty} f^{*}(t) d \xi^{*}(t)
$$

Beweis: Weil $\xi$ und $\xi^{*}$ zusammengeordnet sind, ist jedes Element

$$
z=\int_{-\infty}^{+\infty} f(t) d \xi(t)
$$

in $L_{2}(\xi)$ auch ein Element in $L_{2}\left(\xi^{*}\right)$. Es gibt somit eine quadratisch integrierbare Funktion $f^{*}$ so, dass (36) gilt. Wir sollen beweisen, dass $f^{*}$ die Fouriersche Transformierte von $f$ ist. Nach (34) gilt aber für jedes $S$

$$
E z \overline{\xi(S)}=\int_{S} f(t) d t=\frac{1}{\sqrt{2 \pi}} \int_{-\infty}^{+\infty} f^{*}(\lambda)\left\{\int_{S} e^{-i \lambda t} d t\right\} d \lambda .
$$

Es folgt daraus, dass fast überall

$$
f(t)=\frac{d}{d t}\left\{\frac{1}{\sqrt{2 \pi}} \int_{-\infty}^{+\infty} f^{*}(\lambda) \frac{e^{-i \lambda t}-1}{-i \lambda} d \lambda\right\}
$$

Dies ist aber ein wohlbekannter Ausdruck für die FourIERsche Transformierte der quadratisch integrierbaren Funktion $f^{*}$.

8. Wir sagen, dass eine homogene zufällige Spektralfunktion $\xi(S)$ einer stationären zufälligen Funktion $x(t)$ untergeordnet bzw. zusammengeordnet ist, wenn $\xi\left(T_{t} S\right)$ für jedes $S$ als eine Funktion von $t$ die betreffende Eigenschaft besitzt. Man sieht sofort, dass in (31) $x(t)$ und $\xi(S)$ stationär zusammengeordnet sind.

Es sei $L_{2}(\xi ; t)$ derjenige Unterraum in $L_{2}(\xi)$, der durch alle $\xi(S)$, in welchen $S$ messbare Teilmengen der Halbachse $(-\infty, t)$ sind, aufgespannt wird. Die Elemente von $L_{2}(\xi ; t)$ können in der Form

$$
z=\int_{-\infty}^{t} f(u) d \xi(u)
$$

dargestellt werder, wo $f(u)$ über $(-\infty, t)$ quadratisch integrierbar ist. Wir sagen, dass $x(t)$ und $\xi(S)$ gleichförmig zusammengeordnet $\operatorname{sind}$, wenn $L_{2}(x ; t)=$ $=L_{2}(\xi ; t)$ für jedes $t$ gilt.

Wir fragen nun, ob in $(31) \xi(S)$ so gewählt werden kann, dass sie mit $x(t)$ gleichförmig zusammengeordnet ist. Wir zeigen zuerst, dass, angenommen dass eine solche $\xi(S)$ existiert, sie bis auf einen Zahlenfaktor vom absoluten Betrag 1 eindeutig bestimmt ist.

Es sei also 


$$
x(t)=\int_{-\infty}^{t} g_{1}(t-u) d \xi_{1}(u)=\int_{-\infty}^{t} g_{2}(t-u) d \xi_{2}(u)
$$

und $L_{2}\left(\xi_{1} ; t\right)=L_{2}\left(\xi_{2} ; t\right)=L_{2}(x ; t)$ für jedes $t$. Dann gibt es für jede messbare Menge $S$ eine für $t \in S$ definierte Funktion $f(t ; S)$ so, dass

$$
\xi_{2}(S)=\int_{S} f(t ; S) d \xi_{1}(t)
$$

Wegen $\xi_{2}\left(S_{1}+S_{2}\right)=\xi_{2}\left(S_{1}\right)+\xi_{2}\left(S_{2}\right)$ für $S_{1} \cdot S_{2}=0$ gilt aber

$$
\begin{aligned}
\xi_{2}\left(S_{1}+S_{2}\right)=\int_{S_{1}+S_{2}} f\left(t ; S_{1}+S_{2}\right) & d \xi_{1}(t)= \\
& =\int_{S_{1}} f\left(t ; S_{1}\right) d \xi_{1}(t)+\int_{S_{2}} f\left(t ; S_{2}\right) d \xi_{1}(t),
\end{aligned}
$$

also $f\left(t ; S_{1}\right)=f\left(t ; S_{1}+S_{2}\right)$, wenn $t \in S_{1}$, für alle $S_{2}$. Somit ist $f(t ; S)$ für jedes $t$ von $S$ unabhängig und wir können statt $f(t ; S)$ einfach $f(t)$ schreiben. (37) gibt dann für jedes $t$

$$
x(t)=\int_{-\infty}^{t} g_{1}(t-u) d \xi_{1}(u)=\int_{-\infty}^{t} g_{2}(t-u) f(u) d \xi_{1}(u),
$$

also wegen $L_{2}(x ; t)=L_{2}\left(\xi_{1} ; t\right)$

$$
g_{1}(t-u)=g_{2}(t-u) f(u),
$$

woraus $f(u)=$ Konst. $=k$ folgt. Wir haben somit $\xi_{2}(S)=k \xi_{1}(S)$. Wegen $\left\|\xi_{2}(S)\right\|^{2}=|k|^{2}\left\|\xi_{1}(S)\right\|^{2}=m(S)$, ist $|k|=1$.

Es ist einleuchtend, dass $x(t)$ rein indeterministisch ist, wenn es eine ihr gleichförmig zusammengeordnete $\xi(S)$ existiert. In der Tat: Ist $z$ ein Element in $L_{2}(x ;-\infty)$, so ist es dann auch ein Element in $L_{2}(\xi ; t)$, d. h. in der Form

$$
z=\int_{-\infty}^{t} f(u) d \xi(u)
$$

darstellbar. Diese Darstellung, welche eindeutig ist, muss aber für beliebiges $t$ gelten, was offenbar nur möglich ist, wenn $f(u)$ identisch verschwindet.

9. Um die Existenz der zu $x(t)$ gleichförmig zusammengeordneten $\xi(S)$ zu beweisen, brauchen wir einige analytische Hilfssätze, die teilweise in den Arbeiten von Paley und Wiener [1] sowie Hille und Tamarkin [1, 2] enthalten sind (vgl. auch Nevanlinna [1], S. 174-197).

Hilfssatz 1: Es sei $f(u)$ eine für reelle $u$ definierte komplexwertige und über $(-\infty,+\infty)$ quadratisch bzw. absolut integrierbare Funktion, deren Fouriersche Transformierte $f^{*}(t)$ für $t<0$ identisch verschwindet. Dann wird durch 
K. KARHUNEN, Über die Struktur stationärer zufälliger Funktionen

$$
f(w)=\frac{1}{\sqrt{2 \pi}} \int_{0}^{\infty} e^{-i i w} f^{*}(t) d t \quad(w=u+i v)
$$

eine für $v<0$ reguläre analytische Funktion definiert, die der Bedingung

$$
\int_{-\infty}^{+\infty}|f(u+i v)|^{p} d u<M(f) \text { für alle } v \leqq 0 \quad(p=2, \text { bzw. } 1)
$$

genügt. Hier ist $M(f)$ eine Konstante, die nur von der Wahl der Funktion $f$, aber nicht von $v$ abhängig ist. Umgekehrt kann jede Funktion $f(w)$ mit den letztgenannten Eigenschaften in der Form (39) dargestellt werden, wo $f^{*}(t)$ quadratisch integrierbar, bezw. beschränkt ist. Es gilt weiter

$$
\lim _{v \rightarrow 0} f(u+i v)=f(u) \quad \text { für fast alle } u
$$

und

$$
\lim _{v \rightarrow 0} \int_{-\infty}^{+\infty}|f(u+i v)-f(u)|^{p} d u=0 \quad(p=2 \text { bzw. } 1)
$$

Hilfssatz 2: Eine Funktion $f(w)$, die über die reelle Achse quadratisch bzw. absolut integrierbar ist, erfüllt die Bedingungen des Hilfssatzes 1 dann und nur dann, wenn das Integral

$$
\int_{-\infty}^{+\infty} \frac{|\log | f(t)||}{1+t^{2}} d t
$$

konvergiert und für $v<0$ die Darstellung

$$
\left.f(w)=\Pi_{l}(w) e^{-\frac{1}{\pi i}} \int_{-\infty}^{+\infty} \frac{1+t w}{t-w} \frac{\log |f(t)|}{1+t^{2}} d t+\frac{1}{\pi i} \int_{-\infty}^{+\infty} \frac{1+t w}{t-w} d \chi \chi_{f} t\right)+i \alpha_{f}-i \beta_{f} w
$$

gilt. Hier bedeutet $\Pi_{f}(w)$ ein BlaschKe-Produkt

$$
\Pi_{f}(w)=\prod_{v} \frac{w-w_{v}}{w-\bar{w}_{v}} \frac{\bar{w}_{v}-i}{w_{v}+i}
$$

mit $v_{v}<0$ und

$$
\sum_{v} \frac{v_{v}}{1+\left|w_{v}\right|^{2}}<\infty
$$

$\chi_{f}(t)$ eine beschränkte nichtabnehmende Funktion, deren Ableitung fast überall verschwindet, $a_{f}$ eine reelle Zahl und $\beta_{f}$ eine positive Zahl. Die Darstellung (43) ist eindeutig. 
Den Beweis des Hilfssatzes 1 findet man bei Paley und Wiener [1] für quadratisch integrierbare $f$ und bei Hille und TAmarkin [2] für absolut integrierbare $f$.

Beweis des Hilfssatzes 2: Es sei $f(w)$ durch (43) definiert. Offenbar ist $f(w)$ für $v<0$ analytisch und regulär. Die Funktion

$$
e^{-\frac{1}{\pi i}} \int_{-\infty}^{+\infty} \frac{1+t w}{t-w} \frac{\log |f(t)|}{1+t^{2}} d t
$$

genügt der Bedingung (40) (Paley und Wiener [1], Satz XII). Der Ausdruck

$$
\Pi_{f}(w) e^{\frac{1}{\pi i}} \int_{-\infty}^{+\infty} \frac{1+t w}{t-w} d \chi_{f}(t)-i \beta_{f} w+i \alpha_{f}
$$

ist aber für $v<0$ beschränkt und hat für $v \rightarrow 0$ fast überall beschränkte Randwerte, so dass auch $f(w)$ dieselbe Bedingung (40) erfüllt. - Es sei umgekehrt $f(w)$ eine Funktion; die den Bedingungen des Hilfssatzes 1 genügt. Durch

$$
w=i \frac{z+1}{z-1}
$$

wird $f(w)$ in eine Funktion $g(z)$ transformiert, die innerhalb des Einheitskreises analytisch und regulär ist. Aus (40) folgt weiter, dass

$$
\int_{0}^{2 \pi} \mid g\left(\left.r e^{i(r)}\right|^{p} d \varphi \leqq M_{f} \quad(p=2 \text { bzw. } 1)\right.
$$

für $r \leqq 1^{*}$ (Hille und Tamakkin [2]). "Wird wie gewöhnlich mit $\log ^{+} a$ die grössere der Zahlen $\log a$ und 0 bezeichnet, folgt daraus

$$
\int_{0}^{2 \pi} \log ^{+}\left|g\left(r e^{i \varphi}\right)\right| d \varphi \leqq M_{f}
$$

für $r \leqq 1$. Weil $g(z)$ im Einheitskreise keine Pole hat, ist sie danach eine für $|z|<1$ beschränktartige Funktion (Nevandinsa [1]). Eine solche Funktion kann aber in der Form

$$
g(z)=\Pi(z) e^{\frac{1}{2 \pi} \int_{0}^{2 \pi} \frac{e^{i 9}+z}{e^{i y^{2}-z}} d \psi(9)+i \alpha}
$$

dargestellt werden, wo $\Pi(z)$ ein BLaschke-Produkt und $\psi$ eine Funktion von beschränkter Schwankung ist. Es gilt

$$
\psi(\vartheta)=\lim _{r \rightarrow 1} \int_{0}^{\vartheta} \log \left|g\left(r e^{i \varphi}\right)\right| d \varphi
$$


K. KARHUNEN, Über die Struktur stationärer zufälliger Funktionen

Wenn für einen Wert $\vartheta$ die Ableitung $\psi^{\prime}(\vartheta)$ endlich ist, so ist

$$
\psi^{\prime}(\vartheta)=\lim _{r \rightarrow 1} \log \left|g\left(r e^{i \vartheta}\right)\right| \text {. }
$$

Weil $\psi(\vartheta)$ als eine Funktion von beschränkter Schwankung fast überall eine endliche Ableitung hat, gilt (49) fast überall und die totalstetige Komponente von $\psi(\vartheta)$ ist dadurch bestimmt. Es sei $\psi^{+}$die positive Variation von $\psi$. Aus (48) folgt

$$
\psi^{+}(\vartheta)=\lim _{r \rightarrow 1} \int_{0}^{\vartheta} \log ^{+}\left|g\left(r e^{i \varphi}\right)\right| d \varphi .
$$

Nun gilt aber wegen (42) (vgl. Hille und Tamarkin [2])

$$
\lim _{r \rightarrow 1} \int_{0}^{2 \pi} g\left(r e^{i \varphi}\right) d \varphi=\int_{0}^{2 \pi} g\left(e^{i \varphi}\right) d \varphi
$$

und fast überall $g\left(e^{i} r\right)=\lim _{r \rightarrow 1} g\left(r e^{i \varphi}\right)$, woraus folgt, dass das Integral

$$
\int_{0}^{9}\left|g\left(r e^{i \varphi}\right)\right| d \varphi
$$

für $r<1$ gleichmässig totalstetig ist. Wegen $0 \leqq \log ^{+}|g| \leqq|g|$ ist dann auch

$$
\int_{0}^{9} \log ^{+}\left|g\left(r e^{i \varphi}\right)\right| d \varphi
$$

für $r<1$ gleichmässig totalstetig und somit nach $(50) \psi^{+}(\vartheta)$ totalstetig (vgl. Nevanlinna [1], S. 194). Es folgt daraus, dass die Funktion

$$
\psi_{0}(\vartheta)=\psi(\vartheta)-\int_{0}^{9} \psi^{\prime}(\varphi) d \varphi,
$$

deren Ableitung fast überall verschwindet, nichtzunehmend ist. Weil schliesslich fast überall $\lim _{r \rightarrow 1} \log \left|g\left(r e^{i(r}\right)\right|=\log \left|g\left(e^{i(r)}\right)\right|$ ist, folgt aus (49) und (51)

$$
\psi(\vartheta)=\int_{0}^{\vartheta} \log \left|g\left(e^{i \varphi}\right)\right| d \varphi+\psi_{0}(\vartheta) .
$$

Aus (47), (52) und (46) folgt nun leicht für $f(w)$ die Darstellung (43), wenn man die aus (46) folgende Relation

$$
d \vartheta=\frac{d u}{1+u^{2}}
$$

berücksichtigt und den Sprung von $\psi_{0}$ für $\vartheta=0$ mit $\beta$ bezeichnet. 
Hilfssatz 3: Es sei $f^{*}(t)$ eine für $t>0$ definierte quadratisch integrierbare komplexe Funktion. Hinreichend dafür, dass es keine ebenso für $t>0$ definierte, nicht identisch verschwindende quadratisch integrierbare Funktion $h^{*}(t)$ so gibt, dass

$$
q^{*}(t)=\int_{0}^{\infty} f^{*}(u) h^{*}(u+t) d u=0 \quad \text { für alle } t>0,
$$

ist, dass für die durch (39) definierte Funktion $f(w)$ die Darstellung

$$
f(w)=e^{-\frac{1}{\pi i}} \int_{-\infty}^{+\infty} \frac{1+t w}{t-w} \frac{\log |f(t)|}{1+t^{2}} d t+i \alpha_{f}
$$

gilt. (Wir werden später sehen, dass diese Bedingung auch notwendig ist).

Beweis: Setzt man für $t<0 h^{*}(t)=0$, wird $q^{*}(t)$ auch für $t<0$ definiert; wegen der quadratischen Integrierbarkeit von $t^{*}$ und $h^{*}$ ist sie für alle $t$ beschränkt. Ebenso können wir $f^{*}(t)=0$ für $t<0$ definieren. Wir schreiben ferner

$$
\begin{cases}h(w)=\frac{1}{\sqrt{2 \pi}} \int_{-\infty}^{+\infty} e^{i t w} h^{*}(t) d t \quad \text { für } v>0, \\ q(w)=\frac{1}{\sqrt{2 \pi}} \int_{-\infty}^{+\infty} e^{i t w} q^{*}(t) d t \quad \text { für } v<0 .\end{cases}
$$

Nach dem Hilfssatz 1 gilt fast überall

$$
\lim _{v \rightarrow 0} f(w)=f(u), \quad \lim _{v \rightarrow 0} h(w)=h(u),
$$

wo $f$ und $h$ die Fourierschen Transformierten von $f^{*}$ und $h^{*}$ sind. Die ParsEVALsche Gleichung gibt nach (53)

$$
q^{*}(t)=\int_{-\infty}^{+\infty} e^{-i u t} f(u) h(u) d u
$$

Nach dem Hilfssatz 1 gilt dann, weil $f(t) h(t)$ absolut integrierbar ist,

$$
q(u)=\lim _{v \rightarrow 0} q(u+i v)=f(u) h(u) .
$$

Die Funktion $g(w)$ ist für $v<0$ analytisch und in der Form (43) darstellbar. Weil $h(w)$ für $v>0$ analytisch ist, ist auch $\overline{h(\bar{w})}$ eine für $v<0$ analytische Funktion, die offenbar den Bedingungen des Hilfssatzes 1 genügt und somit in der Form (43) darstellbar ist. Wir haben somit, wenn $f(w)$ die Bedingung (54) erfüllt, 
K. KARHUNEN, Über die Struktur stationärer zufälliger Funktionen

$$
\begin{aligned}
\Phi(w) & =\overline{h(\bar{w})} \frac{q(w)}{f(w)}=\Pi_{h}(w) \Pi_{q}(w) \times \\
& \exp \left\{-\frac{1}{\pi i} \int_{-\infty}^{+\infty} \frac{1+t w}{t-w} \frac{\log |h(t)|+\log |q(t)|-\log |f(t)|}{1+t^{2}} d t+\right. \\
& \left.+\frac{1}{\pi i} \int_{-\infty}^{+\infty} \frac{1+t w}{t-w} d\left(\chi_{h}(t)+\chi_{q}(t)\right)-i\left(\beta_{h}+\beta_{q}\right) w+i\left(\alpha_{h}+\alpha_{q}+\alpha_{f}\right)\right\}
\end{aligned}
$$

Hier ist $\Pi_{h}(w) \Pi_{q}(w)$ ein BLASCHKE-Produkt, $\chi_{h}(t)+\chi_{q}(t)$ ist nichtabnehmend und $\beta_{h}+\beta_{q} \geqq 0$. $\Phi(w)$ ist also in der Form (43) darstellbar und hat fast überall die Randwerte

$$
\Phi(u)=\lim _{v \rightarrow 0} \Phi(u+i v)=\overline{h(u)} \frac{q(u)}{f(u)}=|h(u)|^{2} .
$$

Weil $h(u)$ quadratisch integrierbar ist, ist $\Phi(u)$ absolut integrierbar und hat somit nach den Hilfssätzen 1 und 2 eine FourIersche Transformierte $\Phi^{*}(t)$, die für $t<0$ verschwindet. Nach $(57)$ ist aber $\Phi(u)$ reell und somit $\Phi^{*}(-t)=$ $=\overline{\Phi^{*}(t)}$. Es folgt daraus, dass $\Phi^{*}(t)$ und mit ihr $\Phi(u), h(u)$ und schliesslich $h^{*}(t)$ identisch verschwinden.

10. Es sei $\xi(S)$ eine homogene zufällige Spektralfunktion, für welche (31) gilt. Weil dann jedenfalls $L_{2}(x ; t) \subseteq L_{2}(\xi ; t)$, sind $x(t)$ und $\xi(S)$ dann und nur dann gleichförmig zusammengeordnet, wenn es in $L_{2}(\xi ; t)$ kein zu $L_{2}(x ; t)$ orthogonales Element gibt. Weil jedes Element von $L_{2}(\xi ; t)$ in der Form $(38)$ darstellbar ist, gilt für $u \leqq t$

$$
E(x(u) \bar{z})=\int_{-\infty}^{u} g(u-v) \overline{f(v)} d v=\int_{0}^{\infty} g(v) \overline{f(u-v)} d v .
$$

Ist $z \perp L_{2}(x ; t)$, so muss dieser Ausdruck für alle $u \leqq t$ verschwinden. Wird $\overline{f(t-v)}=h(v)$ bezeichnet, sieht man, dass aus $z \perp L_{2}(x ; t)$

$$
\int_{0}^{\infty} g(v) h(v+u) d v=0
$$

für alle $u>0$ folgt. Es gilt $L_{2}(x ; t)=L_{2}(\xi ; t)$ dann und nur dann, wenn es keine $h(v)$ mit dieser Eigenschaft gibt. Nach dem Hilfssatz 3 ist aber dafür hinreichend, dass für die Funktion

$$
G(w)=\frac{1}{\sqrt{2 \pi}} \int_{0}^{\infty} e^{-i t w} g(t) d t
$$

die Darstellung 


$$
G(w)=e^{-\frac{1}{\pi i}} \int_{-\infty}^{+\infty} \frac{1+t w}{t-w} \frac{\log |G(t)|}{1+t^{2}} d t+i \alpha
$$

gilt. $G(u)$ ist aber die FourIERsche Transformierte von $g(t)$ und genügt somit wegen (31) der Bedingung

$$
|G(\lambda)|^{2}=F_{x}^{\prime}(\lambda) \quad \text { fast überall. }
$$

Wenn wir von dem bedeutungslosen Faktor $e^{i a}$ absehen, ist also

$$
G_{0}(w)=e^{-\frac{1}{2 \cdot i}} \int_{-\infty}^{+\infty} \frac{1+\lambda w}{i-w} \frac{\log F^{\prime} x^{(\lambda)}}{1+\iota^{2}} d \lambda
$$

die einzige $G$-Funktion, für welche (59) gilt. Wird die entsprechende $g$-Funktion mit $g_{0}(t)$ bezeichnet, so gibt es eine homogene zufällige Spektralfunktion $\xi_{0}(S)$ so, dass

$$
x(t) \doteq \int_{-\infty}^{t} g_{0}(t-u) d \xi_{0}(u)
$$

(KARHUNEN [1], S. 72), und $\xi_{0}(S)$ ist mit $x(t)$ gleichförmig zusammengeordnet. Wir wissen schon, dass $\xi_{0}(S)$ und $g_{0}(t)$ eindeutig (bis auf den Faktor $e^{i_{*}}$ ) bestimmt sind. Es folgt daraus insbesondere, dass die hinreichende Bedingung im Hilfssatze 3 auch notwendig ist. ${ }^{1}$

Nach dem Hilfssatz 2 bekommt man alle Funktionen $g(t)$, für welche (31) gilt, wenn man die entsprechenden $G$-Funktionen aus

$$
G(w)=G_{0}(w) \Pi(w) e^{\frac{1}{\pi} i} \cdot \int_{-\infty}^{+\infty} \frac{1+i w}{i-w} d X(\lambda)-i \beta w+i a
$$

bestimmt, wo $\Pi(w), \chi(\lambda), \beta$ und $\alpha$ den im Hilfssatz 2 gesagten Bedingungen genügen sollen, aber sonst beliebig gewählt werden können.

11. Wir wollen schliesslich für die zufällige Spektralfunktion $\xi(S)$ in (31) und insbesondere für $\xi_{0}(S)$ einen Ausdruck konstruieren.

Wegen der vorausgesetzten Totalstetigkeit von $F_{x}$ folgt aus $(60)$, wenn $G(w)$ nach (63) bestimmt ist, dass durch

$$
\dot{\xi}^{*}(S)=\int_{\dot{s}} \frac{d Z_{x}(\lambda)}{G(\lambda)}
$$

\footnotetext{
1 Dies konnte man natürlich auch direkt z. B. so zeigen, dass man eine von Null verschiedene Funktion $h(w)$ konstruiert, was möglich ist, wenn die gesagte Bedingung nicht erfüllt ist. Diese Konstruktion ist aber ziemlich umständlich und wir , wollen sie nicht hier durchfïhren, weil wir das Resultat zu nichts gebrauchen könnon.
} 
K. KARHUNEN, Über die Struktur stationärer zufälliger Funktionen

eine homogene zufällige Spektralfunktion definiert wird. In der Tat ist

$$
\left\|\xi^{*}(S)\right\|^{2}=\int_{S} \frac{d F_{x}(\lambda)}{|G(\lambda)|^{2}}=\int_{S} \frac{d F_{x}(\lambda)}{F_{x}^{\prime}(\lambda)}=m(S) .
$$

Ist $\xi(S)$ die Fouriensche Transformierte von $\xi^{*}(S)$, so folgt aus dem Satz 4

$$
\begin{aligned}
x(t)=\int_{-\infty}^{+\infty} e^{i \lambda t} d Z_{x}(\lambda) & =\int_{-\infty}^{+\infty} e^{i \lambda t} G(\lambda) d \xi^{*}(\lambda)= \\
& =\int_{-\infty}^{+\infty} g(t-u) d \xi(u)=\int_{-\infty}^{t} g(t-u) d \xi(u),
\end{aligned}
$$

weil die Fouriensche Transformierte $g(t)$ von $G(\lambda)$ für $t<0$ verschwindet, wenn $G(w)$ durch (63) definiert ist.

12. Die Resultate von $\mathrm{Nr} .10$ und 11 zusammenfassend bekommen wir den

Satz 5: Eine stetige stationäre zufällige Funktion $x(t)$ ist dann und nur dann rein indeterministisch, wenn ihr Spektrum totalstetig und vollständig ist und das Integral

$$
\int_{-\infty}^{+\infty} \frac{\left|\log F_{x}^{\prime}(\lambda)\right|}{1+\lambda^{2}} d \lambda
$$

konvergiert. Diese Bedingung ist mit derjenigen gleichbedeutend, dass $x(t)$ in der Form

$$
x(t)=\int_{-\infty}^{t} g(t-u) d \xi(u)
$$

darstellbar ist, wo $\xi(S)$ eine homogene mit $x(t)$ zusammengeordnete zufällige Spektralfunktion bedeutet. Man bekommt alle solche Darstellungen, wenn man

setzt, wo

$$
\begin{aligned}
& g(t)=\frac{1}{\sqrt{2 \pi}} \int_{-\infty}^{+\infty} e^{i \lambda t} G(\lambda) d \lambda \\
& \xi(S)=\frac{1}{\sqrt{2 \pi}} \int_{-\infty}^{+\infty}\left\{\int_{S}^{\infty} e^{i \lambda t} d t\right\} \frac{d Z_{x}(\lambda)}{G(\lambda)}
\end{aligned}
$$

$$
G(\lambda)=-\lim _{l \rightarrow 0} G(\hat{\lambda}+i / \mu)
$$

und $G(\lambda+i \mu)=G(w)$ für $\mu<0$ durch (61) und (63) bestimmt ist. $\xi(S)$ kann so gewählt werden, dass sie mit $x(t)$ gleichförmig zusammengeordnet ist, und 158 
die entsprechende Darstellung bekommt man, wenn man $G(w)=G_{0}(w)$ nimmt. Diese letztgenannte Darstellung

$$
x(t)=\int_{-\infty}^{t} g_{0}(t-u) d \xi_{0}(u)
$$

ist bis auf einen Normierungsfaktor $e^{i \alpha}$ ( $\alpha$ reell) eindeutig bestimmt.

Als Korollar bekommen wir sofort den

Satz 6: In der Komponentenzerlegung (24) einer stetigen stationären zufälligen Funktion $x(t)$ sind die Komponenten $x_{2}(t)$ und $x_{3}(t)$ deterministisch, die Komponente $x_{1}(t)$ entweder deterministisch oder rein. indeterministisch je nach dem das Integral

$$
\int_{-\infty}^{+\infty} \frac{\left|\log F_{x}^{\prime}(\lambda)\right|}{1+\lambda^{2}} d \lambda
$$

divergiert oder konvergiert. Weil $x_{1}(t)$ die einzige stationäre Komponente von $x(t)$ ist, die ein vollständiges totalstetiges Spektrum haben kann, ist $x_{1}(t)$ auch die einzige stationäre Komponente von $x(t)$, die rein indeterministisch sein kann. Insbesondere ist jede stationäre Komponente einer rein indeterminischen zufälligen Funktion $x(t)$ deterministisch (wenn wir die triviale Kon!ponente $x(t)$ selbst ausschliessen).

\section{§ 4. Lineare Extrapolation einer stationären zufälligen Funktion}

13. Es sei fortwährend $x(t)$ stetig und stationär. Das Problem der linearen Extrapolation von $x(t)$ ist, ein solches Element $z$ in $L_{2}(x ; s)$ zu finden, dass $\|x(t)-z\|(t>s)$ Minimum wird. Dieses Minimun wird bekanntlich für $z=P_{L, x, s} x(t)$ erreicht. Aus den Sätzen 5 und 6 folgt aber sofort

$$
P_{L_{2}(x, s)} x(t)=x_{2}(t)+x_{3}(t)+\int_{-\infty}^{s} g_{0}(t-\cdots u) d \xi_{0}(u) .
$$

Der Extrayolationsfehler ist also

$$
x(t)-P_{L_{2}(x ; s)} x(t)=\int_{s}^{t} g_{0}(t-u) d \xi_{0}(u)
$$

und sein Normquadrat

$$
D_{x}(t-s)=\left\|\int_{s}^{t} g_{0}(t-u) d \xi_{0}(u)\right\|^{2}=\int_{0}^{t-s}\left|g_{0}(u)\right|^{2} d u .
$$

Die nichtakmehmende Funktion $D_{x}(t)$ ist ein Mass fïr die Ungenauigkeit der Extrapolation. Wenn $D_{x}(t)$ identisch verschwindet, ist $x(\prime)$ deterministisch. 


\section{K. KARHUNEN, Über die Struktur stationärer zufälliger Funktionen}

Man bemerkt, dass wir keine explizite Lösung für das Extrapolationsproblem gegeben haben, weil in dem oben gegebenen Ausdruck für $\xi_{0}$ der ganze Raum $L_{2}(x)$ statt des Unterraumes $L_{2}(x ; s)$ zur Anwendung kommt. Man kann auch eine explizite Iösung konstruieren, wir wollen aber nicht diese Frage hier näher untersuchen, sondern hoffen $\mathrm{zu}$ ihr in einem anderen Zusammenhang zurückkommen zu können.

LITERATURVERZEICHNIS. Cramér, H.: [1] On the theory of stationary random processes. - Annals of Mathematics, 41 (1940). -- - : [2] On harmonic analysis in certain functional spaces. - Arkiv för matematik, astronomi oeh fysik, 28 B (1942). - Doob, J. L.: [1] The elementary Gaussian processes. - Annals of Mathematical Statistics, 15 (1944). - Hanner, O.: [1] Deterministic and non-deterministic stationary stochastic processes. - Arkiv för matematik, 1 (1949). - Hille, E. and Tamarkin, J. D.: [1] A theorem of Paley and Wiener. - Annals of Mathematics, 34 (1933). — _ - [2] On the absolute integr bility of Fourier-transforms. - Fundamenta Mathematicae, 25 (1935). Karhunen, K.: [1] Über lineare Methoden in der Wahrscheinlichkeitsrechnung. - Ann. Ac. Sci. Fennicae, A, I, 37 (1947). Khintchine, A.: [1] Korrelationstheorie der stationären stochastischen Prozesse. - - Mathematische Annalen, 109 (1934). - Kolmogoroff, A.: [1) Stationäre Folgen in einem Hilbertschen Raum (russisch). - Bulletin de l'Université de Moscou, 2 (1941). — - _ [2] Interpolation und Extrapolation von stationären zufälligen Folgen. - Bull. Acad. Sci. URSS, Sér. Math., 5 (1941). - Nevanlinna, R.: [1] Eindeutige analytische Funktionen. - Berlin 1936. - Paley, R. and Wiener, N.: [1] Fourier transforms in the complex domain. - New York 1934, - Wold, H.: [I] A study in the analysis of stationary time series. - Uppsala 1938. 